\title{
Advanced Techniques for Fourier Transform Wavefront Reconstruction
}

\author{
L.A. Poyneer
}

This article was submitted to

Astronomical Telescopes and Instrumentation, Waikoloa, Hawaii, August 22-25, 2002

\section{August 5, 2002}

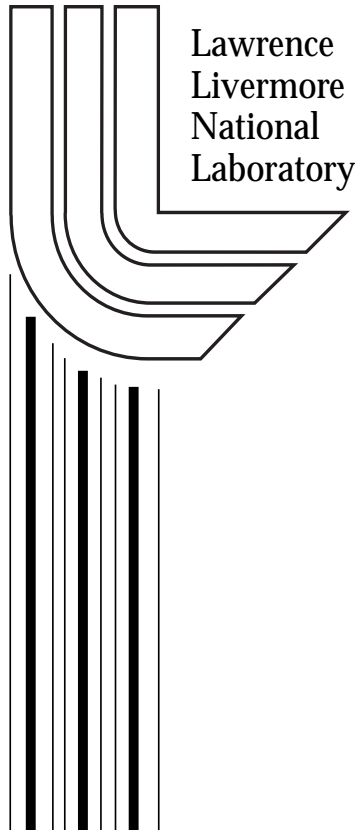




\section{DISCLAIMER}

This document was prepared as an account of work sponsored by an agency of the United States Government. Neither the United States Government nor the University of California nor any of their employees, makes any warranty, express or implied, or assumes any legal liability or responsibility for the accuracy, completeness, or usefulness of any information, apparatus, product, or process disclosed, or represents that its use would not infringe privately owned rights. Reference herein to any specific commercial product, process, or service by trade name, trademark, manufacturer, or otherwise, does not necessarily constitute or imply its endorsement, recommendation, or favoring by the United States Government or the University of California. The views and opinions of authors expressed herein do not necessarily state or reflect those of the United States Government or the University of California, and shall not be used for advertising or product endorsement purposes.

This is a preprint of a paper intended for publication in a journal or proceedings. Since changes may be made before publication, this preprint is made available with the understanding that it will not be cited or reproduced without the permission of the author.

This report has been reproduced directly from the best available copy.

Available electronically at http://www.doe.gov/bridge

Available for a processing fee to U.S. Department of Energy

and its contractors in paper from

U.S. Department of Energy

Office of Scientific and Technical Information

P.O. Box 62

Oak Ridge, TN 37831-0062

Telephone: (865) 576-8401

Facsimile: (865) 576-5728

E-mail: reports@adonis.osti.gov

Available for the sale to the public from

U.S. Department of Commerce

National Technical Information Service

5285 Port Royal Road

Springfield, VA 22161

Telephone: (800) 553-6847

Facsimile: (703) 605-6900

E-mail: orders@ntis.fedworld.gov

Online ordering: http://www.ntis.gov/ordering.htm

OR

Lawrence Livermore National Laboratory

Technical Information Department's Digital Library

http://www.llnl.gov/tid/Library.html 


\title{
Advanced techniques for Fourier transform wavefront reconstruction
}

\author{
Lisa A. Poyneer ${ }^{a}$ \\ ${ }^{a}$ Lawrence Livermore National Lab, 7000 East Ave, Livermore, CA 94550, USA
}

\begin{abstract}
The performance of Fourier transform (FT) reconstructors in large adaptive optics systems with Shack-Hartmann sensors and a deformable mirror is analyzed. FT methods, which are derived for point-based geometries, are adapted for use on the continuous systems. Analysis and simulation show how to compensate for effects such as misalignment of the deformable mirror and wavefront sensor gain. Further filtering methods to reduce noise and improve performance are presented. All these modifications can be implemented at the filtering stage, preserving the speed of FT reconstruction. Simulation of a large system shows how compensated FT methods can have equivalent or better performance to slower vector-matrix-multiply reconstructions.
\end{abstract}

Keywords: Adaptive Optics, Wavefront Reconstruction, Extremely Large Telescopes

\section{INTRODUCTION}

Fourier transform (FT) techniques for wavefront reconstruction have been established for use in large adaptive optics (AO) systems. ${ }^{1}$ This development characterized methods using two discrete models of the sensor geometry. However, important questions remain unanswered: will a model-based reconstructor actually perform well in systems with Shack-Hartmann (SH) wavefront sensors (WFS) and a deformable mirror (DM)? If it does, which of the two FT methods will be better? How can system-dependent factors such as misalignment and sensor gain be corrected for?

All of these questions are addressed in this paper, using a mix of theoretical analysis for idealized cases and Monte Carlo simulations. It shows that plain FT methods, when applied to a complete AO system, can produce significant amounts of residual error. However, these discrepancies can be systematically compensated for. This, along with additional filtering, improves performance to levels comparable to vector-matrix-multiply (VMM) methods.

\section{PREVIOUS FT METHOD RESULTS}

FT reconstructors have already been shown to be workable on large circular apertures and have reasonable noise propagation. The results of this previous work ${ }^{1}$ are summarized here. FT reconstructors are formulated and analyzed for the two common sensor geometry models: the Hudgin geometry and the Fried geometry. In the Hudgin geometry, gradients are the first differences between neighboring actuators. In the Fried geometry, gradients are the average of the two first-differences on a square. Two different FT reconstructors were formulated based on these models as applied to a rectangular SH grid, as shown in Figure 1. These two methods are called Hudgin-FT and Fried-FT. The exact details of the steps involved in these methods are shown in Figure 2

Reconstruction of a circular aperture of data on a square grid involves a boundary problem that preventes accurate reconstruction. This problem is solved by using specific methods to make the gradient sets consistent. These methods involve setting the values of specific gradients outside the aperture in a way to guarantee correct reconstruction inside the aperture. By using these methods FT reconstructors accurately reconstruct all sensed modes inside the aperture.

The motivating factor for investigating FT methods is their speed. Traditional VMM methods compute in $O\left(n^{2}\right)$, where $\mathrm{n}$ is the number of actuators. Computation analysis demonstrates that FT methods are limited

E-mail: poyneer1@ilnl.gov 


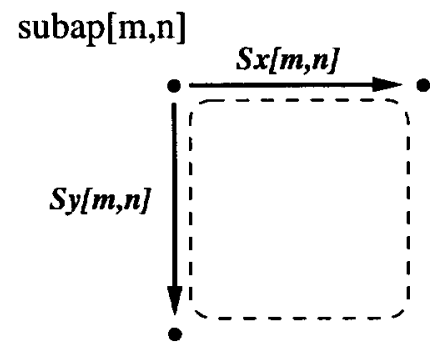

Hudgin

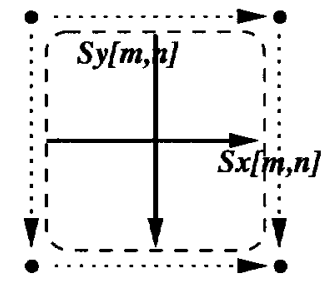

Fried

Figure 1. How the Hudgin- and Fried-geometries are aligned to the rectangular SH sensor array for FT methods. Hudgin-FT assumes the gradients are on the upper and left sides of the square subaperture; Fried-FT assumes that they are the averages of the edges.

in speed only by the Discrete Fourier Transform (DFT) operation. The extra processing to solve the boundary problem is of a lower order of growth computationally. Therefore FFT implementations have computational costs that scale as $O(n \log n)$. However, the implementation of Fried-FT requires potentially 2 times as much total computation as the Hudgin-FT. A 3000 subaperture system can be reconstructed on a $64 \times 64$ grid. This means that the speed of a $64 \times 64$ FFT is the limiting factor for such a large system. This FFT can be calculated on currently available systems in around $1 \mathrm{~ms}$.

Analysis and simulation show that for apertures just smaller than the square reconstruction grid (DFT case), the noise propagations of the FT methods are favorable. For the Hudgin geometry, the noise propagator grows with $O(\ln n)$. For the Fried geometry, the noise propagator is best-fit by a curve that is quadratic in the number of actuators, or $O\left(\ln ^{2} n\right)$. For fixed power-of-two sized grids (required to obtain the speed of the FFT for all aperture sizes) the noise propagator becomes worse when the aperture was much smaller than the grid. The results in the DFT case are shown in in Figure 3 in comparison to established results for VMM reconstructors ${ }^{2,3}$ and Freischlad's work ${ }^{4}$ on FT reconstructors on square apertures.

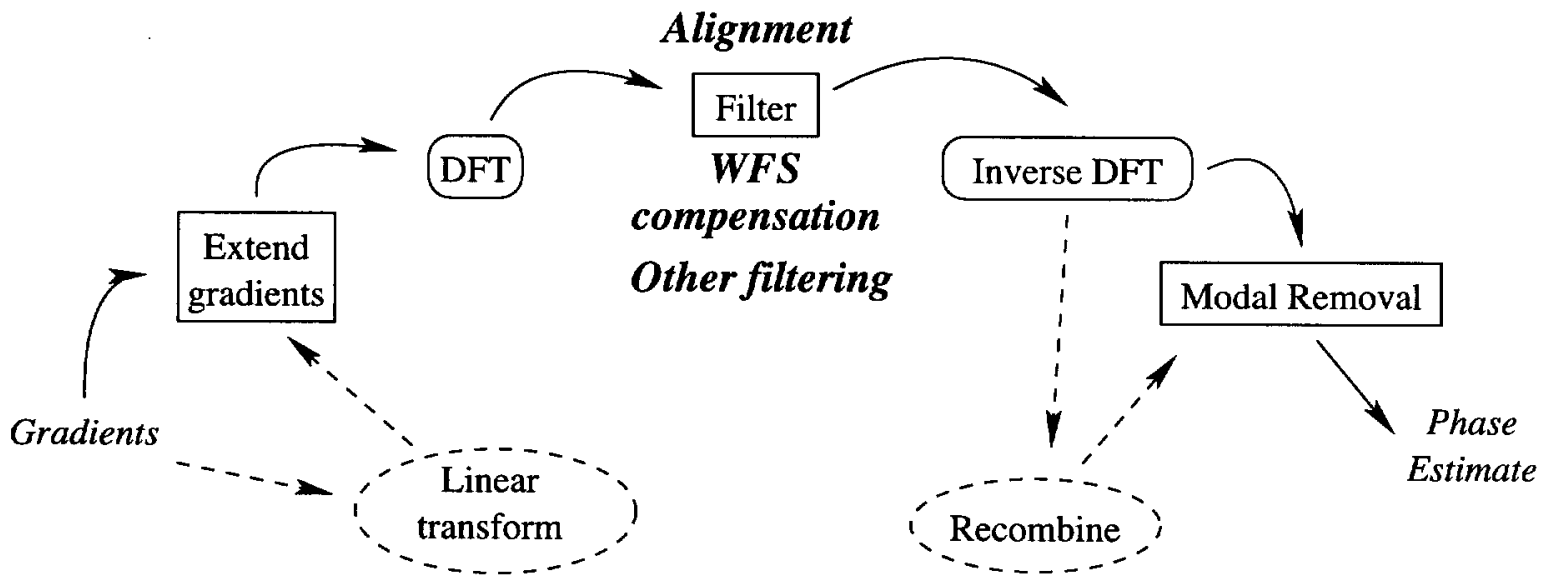

Figure 2. Steps in FT reconstruction. Gradients are extended (to solve the boundary problem), Fourier transformed, filtered, inverse transformed and finally unwanted modes are removed, if necessary. Dashed lines are the extra steps necessary to process the Fried-geometry reconstructor. In bold are the new techniques discussed in this paper to deal with realistic $\mathrm{SH}$ sensors and a DM. 


\section{Noise propagation, Hudgin geometry, DFT}

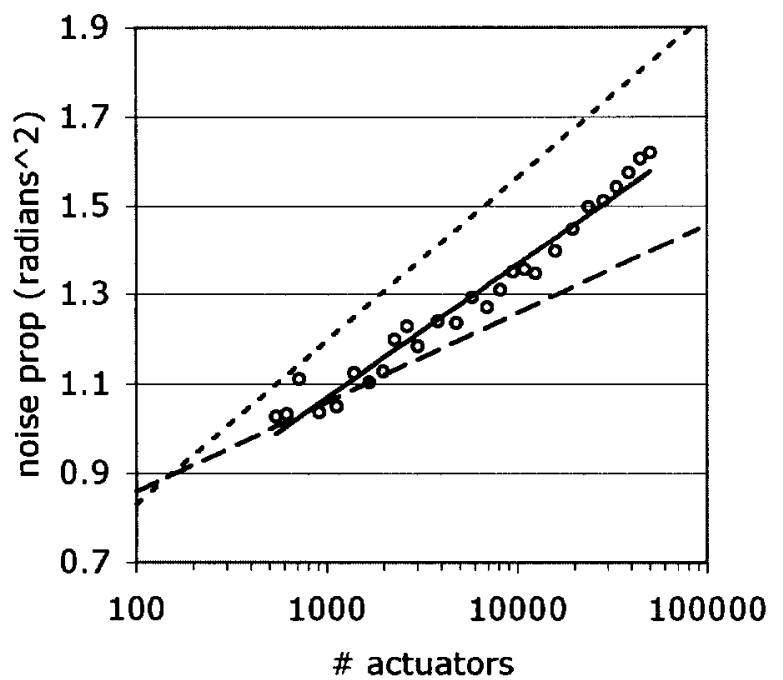

\begin{tabular}{|c|c|}
\hline$\quad$ Hudgin-FT $\cdots \cdots$ \\
$---V M M$ & Freischlad \\
\hline
\end{tabular}

Noise propagation, Fried geometry, DFT

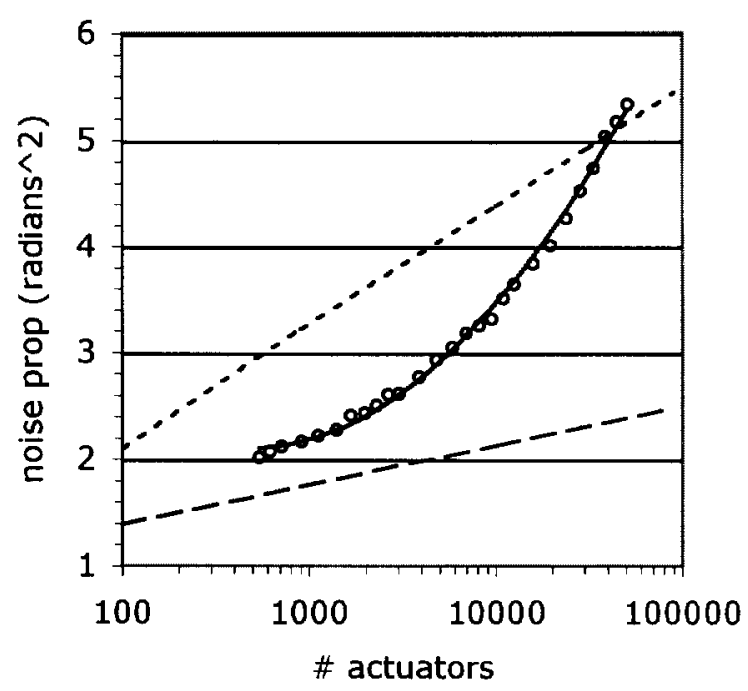

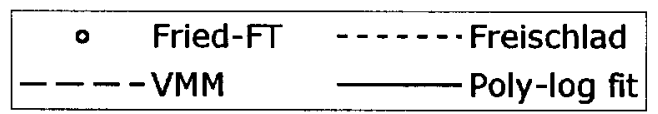

Figure 3. Noise propagation for FT reconstructors, as compared to that for traditional VMM methods ${ }^{2,3}$ and Freischlad's results for square-aperture FT reconstructors ${ }^{4}$ on the same geometries. For apertures slightly smaller than the reconstruction grid, noise propagation is favorable, though higher than for VMM methods.

\section{FT METHODS WITH REALISTIC SH SENSORS AND A DM}

The features of SH sensors and the DM must be included in the performance analysis of FT methods. Previously, covariance-matrix based approaches ${ }^{1}$ were used. However, introducing highly-sampled SH sensors and DM so increases the size of the covariance matrices as to make these computations unreasonable. Instead, signal processing methods will be used to assess performance.

\subsection{Model of open-loop reconstruction}

In order to do a full analysis of the reconstruction process using the standard signal processing techniques of power-spectral densities (PSDs) and transfer functions, the phase disturbance input must be bandlimited and stationary. In addition, the entire reconstruction process must be spatially invariant. In the general atmospheric case, open-loop AO using a FT method meets neither of these criteria. The apertured, pistonremoved phase is neither stationary or bandlimited. The reconstruction process on a circular aperture is not spatially invariant, due to the processing to solve the boundary problem. The process changes the values of certain specific gradients, and is hence not spatially invariant. Reasonable approximations, however, can give insight into actual circular-aperture performance. We seek to find qualitatively useful approximations that aid in understanding performance and design, as opposed to accurate quantitative performance predictions.

For this purpose, the following model is used to explore the properties of FT reconstruction in a realistic open-loop AO system. Parts of the treatment are similar to work published by Rigaut on modeling closed-loop AO systems. ${ }^{5}$ The system has an infinite aperture, and the continuous phase disturbance has a band-limited PSD. In order for the calculations to work, the band-limit is $f=1 / 2 d$, where $d$ is the subaperture width. We will assume von Karman turbulence, which is a modified Kolmogorov spectrum such that the variance of an 
individual point (and hence the over-all mean-squared-error (mse) of the wavefront) is finite. In terms of spatial frequencies $f_{x}, f_{y}$, the power spectrum of this stationary process is ${ }^{6}$ :

$$
W\left(f_{x}, f_{y}\right)=0.49\left(r_{0}\right)^{-5 / 3}\left((2 \pi)^{2}\left(f_{x}^{2}+f_{y}^{2}+\left(1 / L_{0}\right)^{2}\right)\right)^{-11 / 6}
$$

where $L_{0}$ is the outer scale in meters. The SH sensors take the average of the gradient over each subaperture of width $d$. These values are then sampled on a finite grid. Because of our numbering convention relating the subapertures to the finite actuator grid, the averaging function is actually shifted by half an actuator. This part is essential to capturing the true effects of the Hudgin and Fried models being approximations to the real sensors. (See Figure 1 for a clear illustration of this.) In the case of the x-gradients, the slope-taking part has a frequency response of:

$$
S_{x}\left(f_{x}, f_{y}\right)=j 2 \pi f_{x}
$$

The averaging, a convolution with a square window, has a frequency response of:

$$
A_{x}\left(f_{x}, f_{y}\right)=\frac{\sin \left(\pi d f_{x}\right) \sin \left(\pi d f_{y}\right)}{\pi^{2} d^{2} f_{x} f_{y}} \times \operatorname{Exp}\left[\pi d\left(f_{x}+f_{y}\right)\right]
$$

To get the set of discrete measurements, the random process (and hence its covariance function) are sampled every $d$. This scales and replicates the power spectrum. The reconstruction is done with either of the two FT methods, derived for the infinite aperture case. These are discrete filters, so the frequency variables are $\phi_{x}=d f_{x}, \phi_{y}=d f_{y}$ For example, the two parts of the Hudgin-FT are applied to the $\mathrm{x}$ - and y-gradients:

$$
R_{x}\left(\phi_{x}, \phi_{y}\right)=\frac{\left(\operatorname{Exp}\left[-j 2 \pi \phi_{x}\right]-1\right)}{4\left(\sin ^{2} \pi \phi_{x}+\sin ^{2} \pi \phi_{y}\right)}, R_{y}\left(\phi_{x}, \phi_{y}\right)=\frac{\left(\operatorname{Exp}\left[-j 2 \pi \phi_{y}\right]-1\right)}{4\left(\sin ^{2} \pi \phi_{x}+\sin ^{2} \pi \phi_{y}\right)}
$$

After up-sampling the estimate, a low-pass filter corresponding to the DM influence function is applied. For now, ideal low-pass filtering will be assumed.

The residual error is the random process of interest, so it needs to be expressed in terms of a spatiallyinvariant system applied to the phase random process. For the band-limited input case, an exact filter can be derived. It is, assuming an ideal DM :

$$
1-\left[R_{x}\left(d f_{x}, d f_{y}\right) A_{x}\left(f_{x}, f_{y}\right) S_{x}\left(f_{x}, f_{y}\right)+R_{y}\left(d f_{x}, d f_{y}\right) A_{y}\left(f_{x}, f_{y}\right) S_{y}\left(f_{x}, f_{y}\right)\right]
$$

To compute the PSD of the residual error, take the squared-magnitude of the filter and multiply by the PSD of the input phase disturbance. Assuming zero-mean phase disturbance, the variance of the residual error is the integral of the PSD. For the simple case of an ideal low-pass DM, the band-limited frequency response of the system using Hudgin-FT reconstruction is:

$$
E\left(f_{x}, f_{y}\right)=\left|1-\frac{j 2 \sin \left(\pi d f_{x}\right) \sin \left(\pi d f_{y}\right) \operatorname{Exp}\left[\pi d\left(f_{x}+f_{y}\right)\right]\left(\frac{\operatorname{Exp}\left[-j 2 \pi d f_{x}\right]-1}{\pi d^{2} f_{y}}+\frac{\operatorname{Exp}\left[-j 2 \pi d f_{y}\right]-1}{\pi d^{2} f_{x}}\right)}{4\left(\sin ^{2} \pi d f_{x}+\sin ^{2} \pi d f_{y}\right)}\right|^{2} W\left(f_{x}, f_{y}\right)
$$

This analysis can be carried out for both the Fried-FT and Hudgin-FT reconstructors, for a variety of subaperture sizes and DM influence functions. These results, and their interpretation, are covered next.

\subsection{Results for latent error}

Assuming an ideal DM, the PSDs of the residual errors provide insight into the Hudgin-FT and Fried-FT methods. The PSDs in the correctable band of the DM $\left(\left|f_{x}\right|,\left|f_{y}\right|<1 / 2 d\right)$ are shown in Figure 4. The residual error for the two cases has power at quite different spatial frequencies. For the Hudgin-FT, power is concentrated in two peaks at low frequencies. The error for Fried-FT, in contrast, is concentrated around the highest spatial frequency $f_{x}, f_{y}= \pm 1 / 2 d$ (the waffle mode.) This implies that Fried-FT will be more susceptible to aliasing errors, since these will be greatest at high frequencies. These errors are due to the mis-match of the discrete sensor models to real SH behavior. 
That SH sensors take the average gradient is well-established. ${ }^{7}$ Modeling the DM, however, is not so clear-cut a case. In practice, different functions, including Gaussians, have been used as influence function models. ${ }^{7}$ Therefore we will do the same analysis as above, but assuming a non-ideal DM response. The two specific models that we will use are the difference of gaussians model (DG), which is widely used at our lab in simulations. The second model which is a truncated sinc function times a gaussian (SG), which has also been proposed (Kai LaFortune, personal communication.) The frequency response of both of these can be calculated numerically. Both of these DMs produce non-ideal low-pass filtering. The frequency responses, inside the $1 / 2 d$ cutoff, are shown in Figure 5.

The impact of using these non-ideal DMs is clear: power at high frequencies in the phase estimate will be attenuated. As shown in Figure 6, there are two main impacts: the residual error has increased power at low frequencies, and reduced power at high frequencies. This improves the performance of Fried-FT, as its error is at high frequencies, and degrades the performance of Hudgin-FT.

Based on numerical calculations of the residual error in the SG case, Fried-FT has only $14 \%$ of the residual mse of the Hudgin-FT method. This ratio is higher for $d<0.2 \mathrm{~m}$. Monte carlo simulations over various sizes of circular aperture with $d=0.2 \mathrm{~m}$ produce an average ratio of $54 \%$ for high SNRs.

Both this analysis and Monte Carlo simulation results indicate that in terms of latent mse (error due not to noise but to measuring and fitting the wavefront) the Fried-FT has the advantage. Results for the ideal DM case are about equivalent, but for non-ideal DMs, Fried-FT takes a clear edge. However, as shown in Figure 3, Hudgin-FT has a much better noise propagation. In low SNR conditions, error due to noise will dominate the latent error due to the wavefront, and therefore Hudgin-FT would be preferable.

\subsection{Results for noise propagation}

The same kind of PSD analysis can be applied to the problem of noise propagation. In this case, the noise is modeled as stationary white noise on the sampled gradients. This allows for direct evaluation of the resulting signal on the DM. If the input noise is of variance 1, then the sum of the PSD after reconstruction (but before application to the DM) is simply the noise propagation of the reconstruction. After application to the DM, this result can be re-calculated. If an ideal DM is used, the noise propagation is exactly the same across the entire DM surface as it is at just the actuators. The PSDs of the noise for Hudgin-FT and Fried-FT are shown in Figure 7.
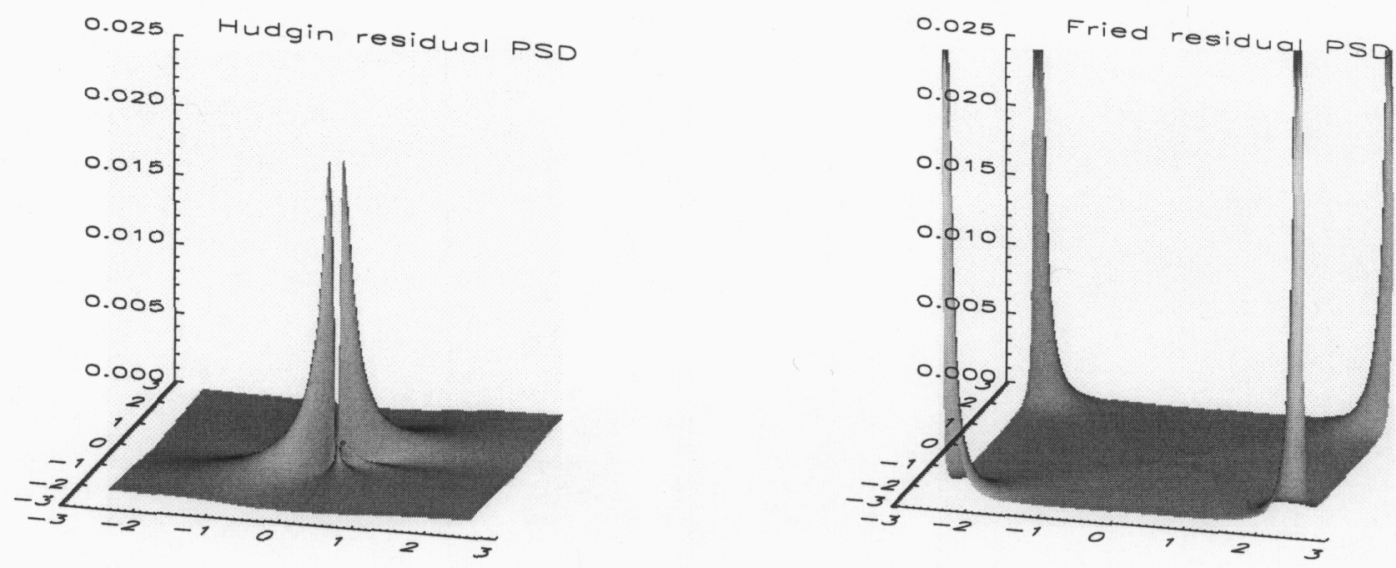

Figure 4. PSDs of the residual error for both FT reconstruction geometries. Calculated with an infinite aperture, $d=0.2 \mathrm{~m}$ and $r_{0}=0.2 \mathrm{~m}$. The residual error for the Hudgin geometry method has power primarily at low frequencies. The Fried-geometry method has error mainly around the waffle-frequency, which is more sensitive to aliasing. PSDs are shown within the pass-band for the ideal DM, i.e. from $-1 / 2 d$ to $1 / 2 d$ in frequency. 

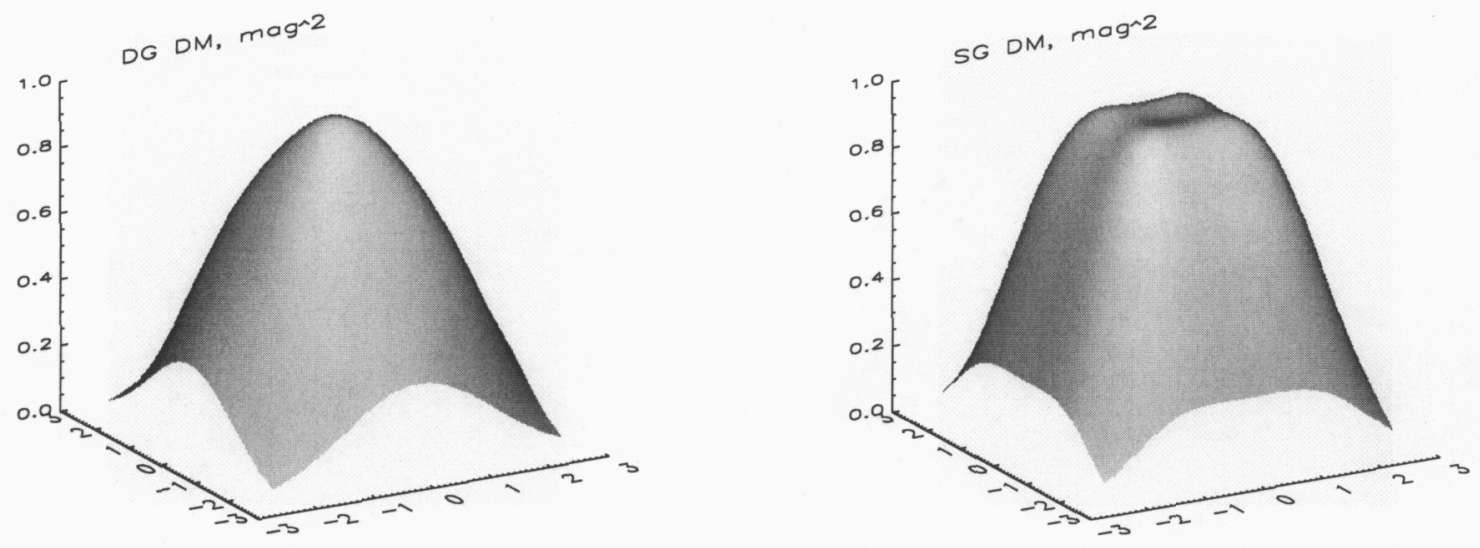

Figure 5. Squared magnitude of the frequency responses of the two non-ideal DM models, DG and SG. The DG model, the difference of two gaussians, is strongly sloped compared to an ideal DM and attenuates high frequencies. The SG model has a broader top and attenuates less than DG at low frequencies. For comparison, an ideal DM would have constant value 1 for the range of plotted frequencies, up to $1 / 2 d$.

This time both methods have power at low-frequencies, and Fried-FT still produces power at high frequencies. As shown in the last subsection, a non-ideal DM will attenuate noise power at high frequencies. The highfrequency components of the noise PSD as shown in Figure 7 will be greatly reduced. This leads to a significant reduction in noise propagation for Fried-FT. Numerical analysis of the PSDs show that for both the SG and DG cases there is a drop in noise propagation. Let $\mathrm{np}_{a c t u}$ indicate the noise propagation calculated at the actuators. Then for the DG case, the noise propagation for the Hudgin-FT is

$$
\mathrm{np}=0.9615 \times \mathrm{np}_{a c t u}-0.1315
$$

and for Fried-FT is:

$$
\mathrm{np}=0.5627 \times \mathrm{np}_{a c t u}+0.0053
$$
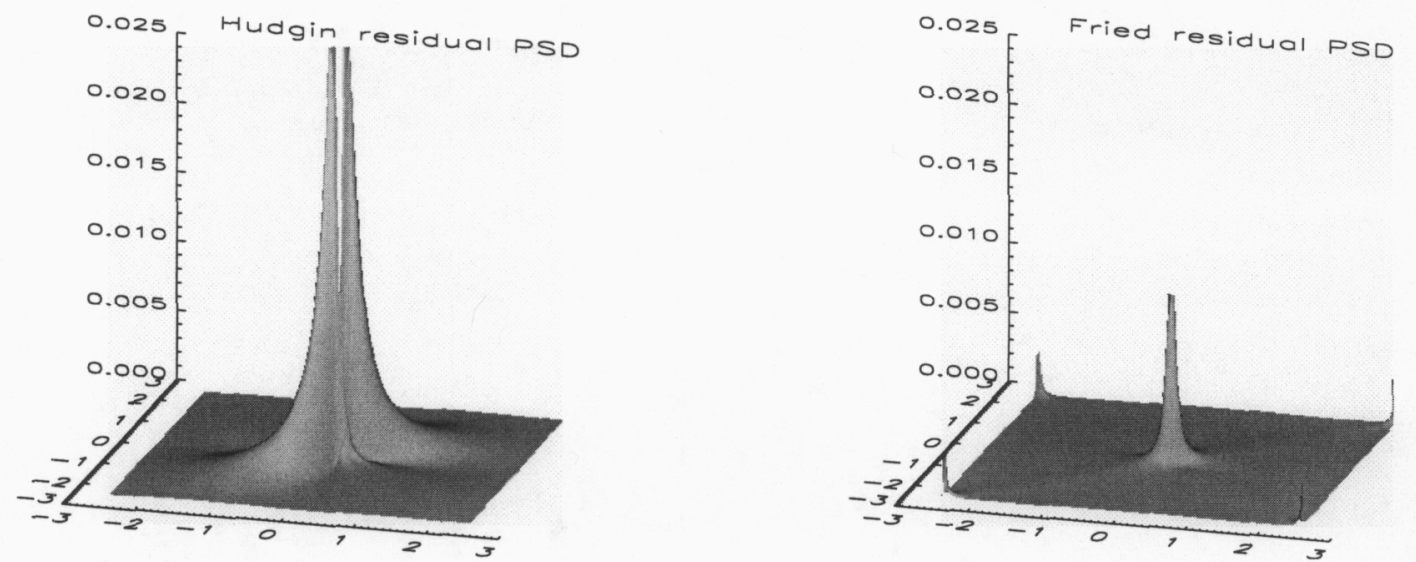

Figure 6. PSDs of the residual error for both FT reconstruction geometries. Calculated as in Figure 4, except that the SG DM model was used. SG increases error at low frequencies and attenuates at high frequencies. The net result is that the Hudgin-FT performs much worse than in the ideal DM case, and Fried-FT performs much better. 
For the SG case the results are for Hudgin-FT:

$$
\mathrm{np}=0.9613 \times \mathrm{np}_{a c t u}-0.0834
$$

and for Fried-FT:

$$
\mathrm{np}=0.6035 \times \mathrm{np}_{a c t u}+0.0503
$$

This means that the noise propagation in the Fried-FT case is reduced by $40 \%$ for the SG DM and by $43 \%$ in the DG DM case. Hudgin-FT has a significantly smaller reduction of roughly $4 \%$.

These results are for the infinite aperture case. Monte carlo simulations for various (finite) sizes of large circular aperture were conducted to determine the reduction in noise propagation in comparison to the infinite aperture case. The results of these simulations are shown in Figure 8. These circular-aperture results are well-correlated with the values predicted by the ideal square-aperture analysis.

This decrease in noise propagation, if a real phenomenon with physical DMs, is highly significant. It cuts the noise propagation for Fried-FT nearly in half. Investigation of this noise reduction is called for both in general studies of DMs and in the design of any specific AO system that will use an FT method.

\section{DEALING WITH OTHER SYSTEM FACTORS}

\subsection{Misalignment}

Besides not capturing the WFS and DM details in the reconstructor, the FT reconstructor does not capture any information about the alignment between the SH sensors and the DM. This misalignment, modeled as linear shifts of the DM in the $\mathrm{x}$ - and $\mathrm{y}$-directions, can easily occur. Presence of misalignment raises two key concerns. First, how much misalignment can be tolerated before there is a significant increase in error? Second, if the misalignment is known, can it be compensated for in the FT reconstructor?

\subsubsection{Magnitude of error due to misalignment}

Misalignment can be introduced directly into the open-loop PSD model. It is simply a shift of the averaging window of the SH sensors. This analysis was conducted for shifts of a variety of magnitudes. The shift of the actuators to the "right" on the grid is defined as positive $\Delta_{x}$; the shift of the actuators "down" on the grid is
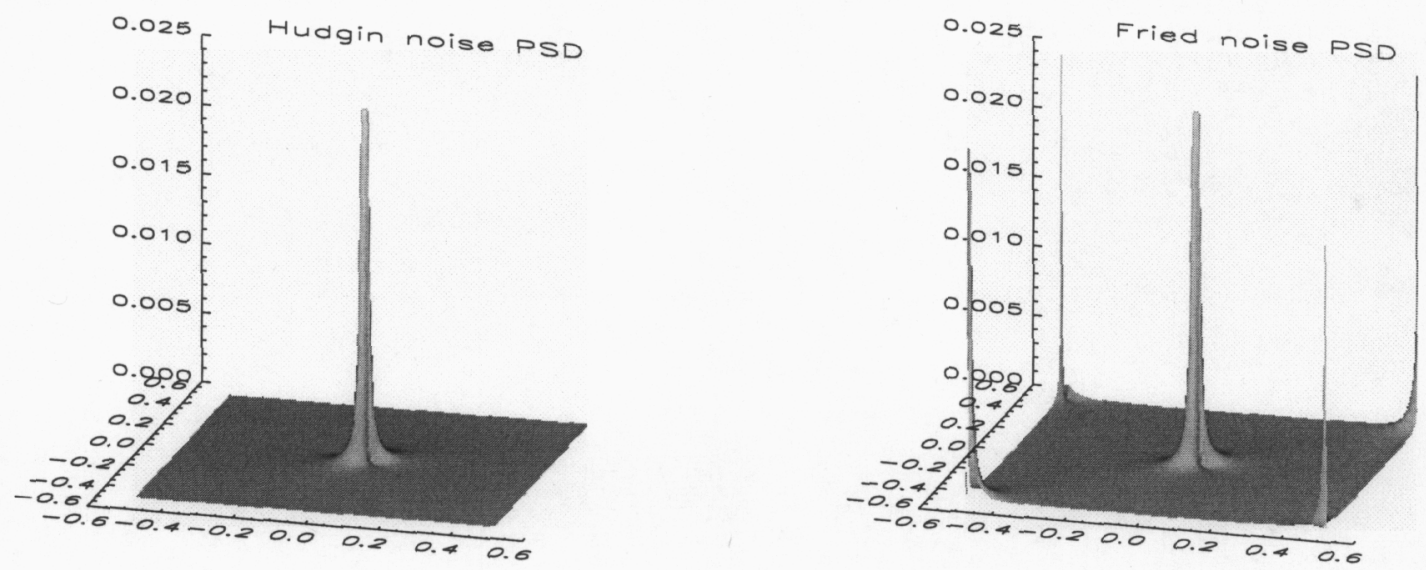

Figure 7. PSDs of the noise for both FT reconstruction geometries, shown for an ideal low-pass DM. In this case the gradients are white noise. Comparing with Figure 4, both methods produce low-frequency noise, and Fried-geometry still has peaks around the waffle frequency. PSDs are shown within the pass-band for the ideal DM. 


\section{Reduction in noise propagation}

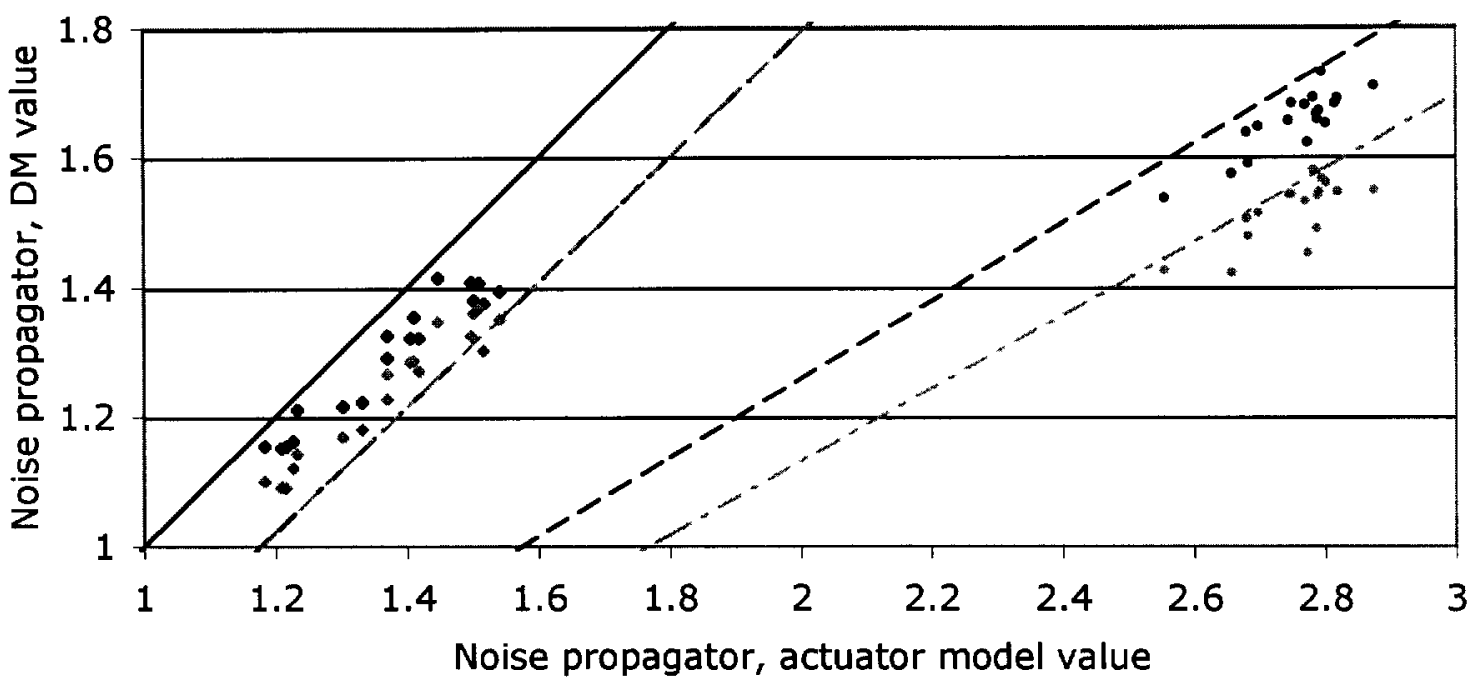

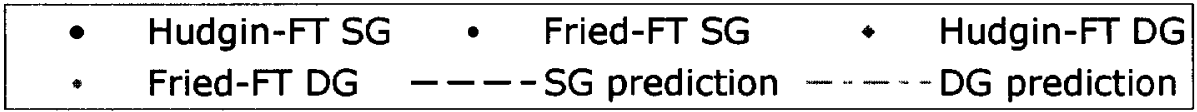

Figure 8. Reduction in noise propagation for a non-ideal DM. The dashed lines are the relationships derived from PSD analysis for infinite apertures. The data points are the results of Monte Carlo simulations on 18 different sizes of large circular apertures. In the circular-aperture case, Fried-FT has a significant reduction in noise propagation of approximately $45 \%$ from the ideal DM case.

defined as positive $\Delta_{y}$. (By numerical convention the origin of the numbering of actuators is in the upper left corner of the aperture.) Explicitly, the windowing function now becomes

$$
A_{x}\left(f_{x}, f_{y}\right)=\frac{\sin \left(\pi f_{x} d\right) \sin \left(\pi f_{y} d\right)}{\pi^{2} d^{2} f_{x} f_{y}} * \operatorname{Exp}\left[\pi d\left(f_{x}+f_{y}\right)\right] * \operatorname{Exp}\left[-2 \pi d\left(\Delta_{x} f_{x}+\Delta_{y} f_{y}\right)\right]
$$

For Fried-FT, these shifts increase the residual error, in a way directly dependent on the magnitude of the total shift $\left(r=\sqrt{\Delta_{x}^{2}+\Delta_{y}^{2}}\right)$. For $d=r_{0}=0.2 \mathrm{~m}$, a shift of magnitude $r=0.175 d$ doubled the residual mse of Fried-FT. The angle of the shift $\left(\theta=\tan ^{-1} \Delta_{y} / \Delta_{x}\right)$ has minimal impact on Fried-FT. This is because the Friedgeometry gradients are effectively centered over the subaperture, so shifts in either direction are symmetric in behavior. For Hudgin-FT, the story is quite different. Misalignment in some cases improved residual error. This is because the $\mathrm{x}$ - and $\mathrm{y}$-gradients in the Hudgin geometry are on the upper and left edges of the subaperture. Shifting the actuators down and to the right actually improves the correlation of the SH sensor measurement to the model, and reduces residual error. (See Figure 1.)The maximum improvement occurred at $\theta=\pi / 4$, that is positive $\Delta_{x}=\Delta_{y}$. The most significant loss of performance occurred when the shift was in exactly the opposite direction. Then the gradients are moving farther away from the section of the phase that they are correlated with. The Hudgin-FT method does not have symmetric behaviour, which has been confirmed by both PSD analysis and simulation. These results are shown in Figure 9.

\subsubsection{The filtering solution to misalignment}

If the misalignment is known, it can be removed by application of a filter in the frequency domain as the phase estimate is computed. This filter can be derived from FT properties and sampling theory (in the general 


\section{Residual mse due to misalignment, \% of original}

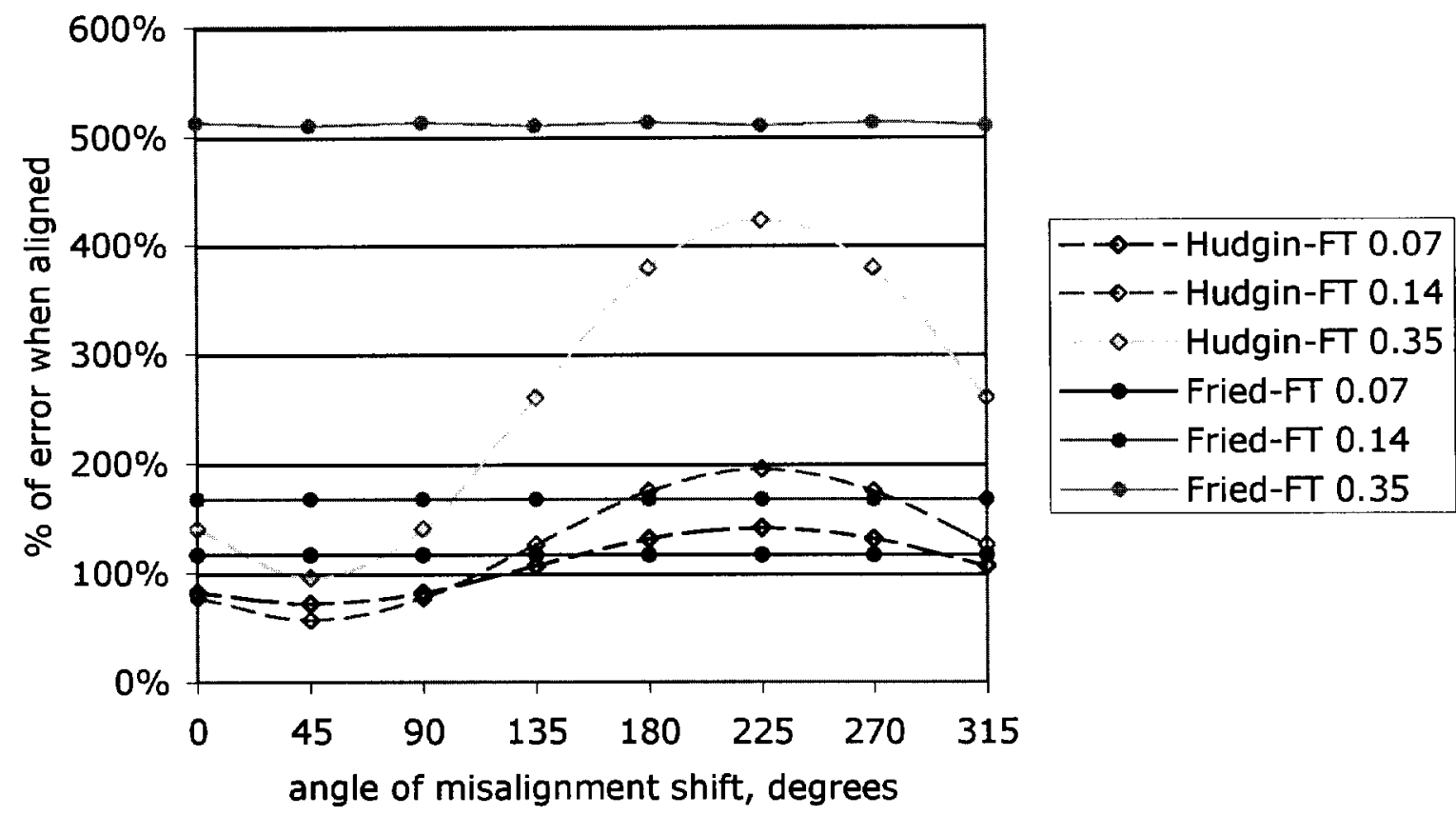

Figure 9. Residual mse when the system is misaligned, as percent of aligned mse. Three sets of misalignments are plotted: $r=0.07 d\left(\max \Delta_{x},=\Delta_{y}=0.05 d\right) ; r=0.14 d\left(\max \Delta_{x}=\Delta_{y}=0.1 d\right) ; r=0.35 d\left(\max \Delta_{x}=\Delta_{y}=0.25 d\right.$. Plotted for $d=r_{0}=0.2 \mathrm{~m}$ and an ideal DM. Two important phenomena are shown. First, misalignments of a tenth of a subaperture in each direction can increase residual mse by two-thirds. Second, Hudgin-FT is highly sensitive to the angle of the shift, with small shifts at angle $\pi / 4$ improving the performance and shifts at $5 \pi / 4$ reducing the performance.

case.) A shift of less than a pixel can be interpreted as the result of upsampling and interpolating to get the band-limited continuous signal, shifting it by less than a sampling interval, and re-sampling it.

If the $N \times N$ grid is mis-aligned in one direction by $\Delta_{x}$, the filter to compensate is: phase:

$$
A[k, l]= \begin{cases}\operatorname{Exp}\left[\frac{j 2 \pi k \Delta_{x}}{N}\right] & k<N / 2 \\ \operatorname{Exp}\left[\frac{j 2 \pi(k-N) \Delta_{x}}{N}\right] & k \geq N / 2\end{cases}
$$

So if the misalignment is known, the re-alignment can be done in a filtering step in the frequency domain. This filtering in practice gives very good results. In the general case there is a tiny increase in estimate error, due to "ringing" that is introduced at the edges. If one end of the finite signal is discontinuous with the other side (due to periodicity for the DFT), there is not enough high-frequency content to fully fit the sharp transition. Therefore there will be artifacts at the edges.

The filtering to correct for alignment errors is in implemented for both the Fried-FT and Hudgin-FT methods. For both it is done in the frequency domain in the same step as the reconstruction filter. Provided the amount of misalignment is known, this filtering process produced very good results. If the amount of misalignment is not exactly known, this method can still be used. Because of the fast implementation at the filtering stage, the amount of misalignment could be easily adjusted in real-time via an interface to the control system. It could be adjusted, just as the loop gain is, until the loop closes with desired performance.

Based on Monte Carlo simulations, the effect of misalignment on noise propagation is minimal, even for large shifts. The PSD analysis of Section 3.3 indicates that there will be no change in noise propagation for alignment 
filtering. Monte carlo simulations showed that for a variety of shifts of $r=0.1 d$, the noise propagation on a large circular aperture varies by less than $2.5 \%$ from the noise propagation with no alignment correction.

\subsection{SH sensor gains}

In practice, a SH sensor produces a measurement which deviates from the exact wavefront slope. This response can be plotted as the measurement versus the actual tilt across the subaperture. The exact shape of this curve depends on number of pixels used per subaperture and the centroid computation method. See Hardy, ${ }^{8}$ section 5.3.1, for a representative set of response curves. The most important feature of the response curve is that even within the linear response range, the gain of the sensor is not unity.

A VMM control matrix derived from an actual AO system would have built-in calibration to this gain factor. An FT reconstructor would not explicitly account for it. Assuming that the gain is uniform across all subapertures, the linearity of the FT reconstructor allows the gain to be compensated for in a single step: a scaling of the estimate. This can be built into the filtering stage. For example, if the gain of the SH sensor for a given system is measured to be 1.25 , the reconstructor would be scaled by 0.8 (which is $1 / 1.25$ ) to produce the correct estimate.

This gain is probably the most important factor to correct for in open-loop. A small error in the gain factor can result significant increase in error. In closed loop this problem in mitigated by the overall control loop gain, which can be adjusted instead.

\section{IMPLICATIONS FOR FILTERING}

The preceding analysis points towards two promising methods of filtering to improve the performance of the FT methods of reconstruction, one for Fried-FT, the other for Hudgin-FT.

For Fried-FT, Section 3 demonstrates how low-pass filtering can reduce both its residual error due to the phase, and its noise propagation. The noise propagation results are confirmed by simulation for the circularaperture case. In a real system, the physical DM should be thoroughly tested to determine its transfer function. Then additional low-pass filtering can be applied to the estimate as necessary to further reduce errors to levels as demonstrated in Section 3.

For Hudgin-FT, re-alignment can improve residual error performance. As discussed in Section 4.1, introducing a misalignment factor can actually improve Hudgin-FT performance. It does so by shifting the model slightly to be more correlated with the actual SH sensor measurements. Use of the ideal PSD model indicates that best shifts are of equal $\Delta_{x}, \Delta_{y}$ and of magnitude $0.175 d<r<0.1827 d$ for a range of $d$ from $0.1 \mathrm{~m}$ to $0.4 \mathrm{~m}$.

\section{SIMULATION RESULTS IN COMPARISON WITH VMM METHODS}

The preceding sections have shown how FT methods perform in an open-loop AO system using analysis and simulation. This section presents a specific result from the realistic open-loop simulations, comparing the performance of plain FT methods to compensated methods and a traditional VMM reconstructor. This comparison is done on a large system with 2,128 subapertures and 2,233 actuators. For a system this size the speed gain from the FT methods is significant. In the present IDL 5.4 implementation on an SGI, calculating the full VMM takes about of $400 \mathrm{~ms}$, while a single FFT of the data (the dominant term in the time of reconstruction) takes $1.3 \mathrm{~ms}$.

In this case both $d$ and $r_{0}$ are set to be $0.2 \mathrm{~m}\left(r_{0}\right.$ measured at $500 \mathrm{~nm}$.) This corresponds to a telescope of diameter of $10.4 \mathrm{~m}$. The VMM control matrix is derived by pushing each DM actuator in turn and measuring the WFS slopes. This 'push' method is used in real VMM AO systems. ${ }^{9}$ The DM is misaligned from the sensors by $\Delta_{x}=-0.1 d, \Delta_{y}=0.05 d$. The DM has a non-ideal SG influence function.

Two comparisons are done. First, the FT methods are applied with no modifications. As shown in Figure 10, performance of both uncompensated FT methods is worse than the VMM. Then fully compensated FT methods are applied to the same WFS data. The WFS gain is compensated for, and filtering is used to correct for the known misalignment. Hudgin-FT also has extra filtering to improve alignment, as described in Section 5 . These 


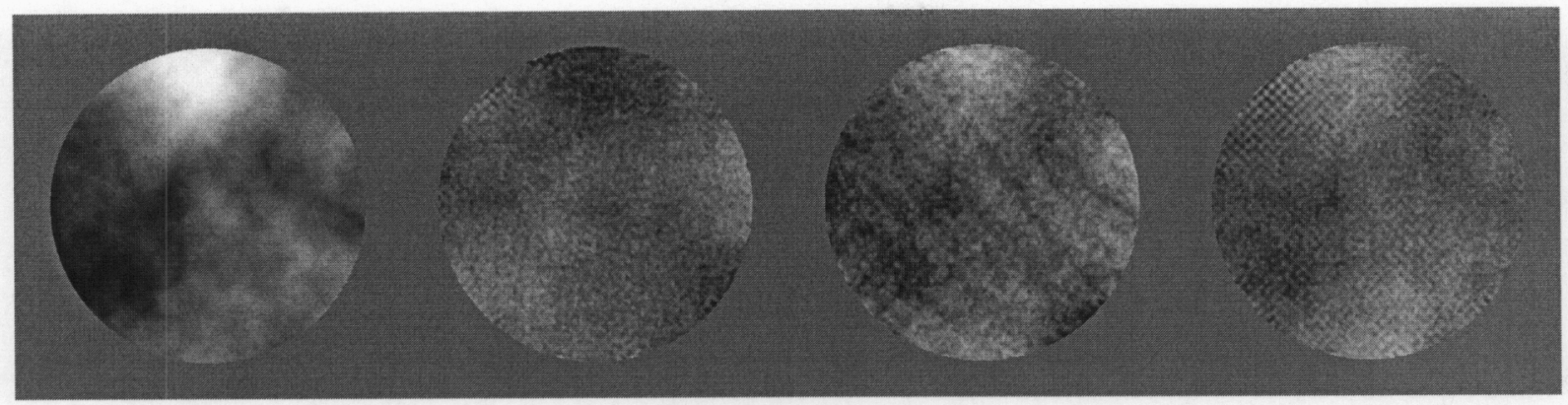

Figure 10. Phase distortion (plotted with a different color scale) compared to residual error, for open-loop VMM and uncompensated Hudgin-FT and Fried-FT methods. Results for $\lambda=1$ micron. Wavefront mse is 108.458 radians $^{2}$; VMM residual mse is 0.413 and Strehl is 0.66 . Uncompensated Hudgin-FT has mse of 0.711, Strehl of 0.49. Uncompensated Fried-FT has mse of 0.542 , Strehl of 0.58 . System parameters: $D=10.4 \mathrm{~m}, d=0.2 \mathrm{~m}, r_{0}=0.2 \mathrm{~m}($ at $\lambda=500 \mathrm{~nm}$.) 2128 subapertures, 2233 actuators. SNR of 12.

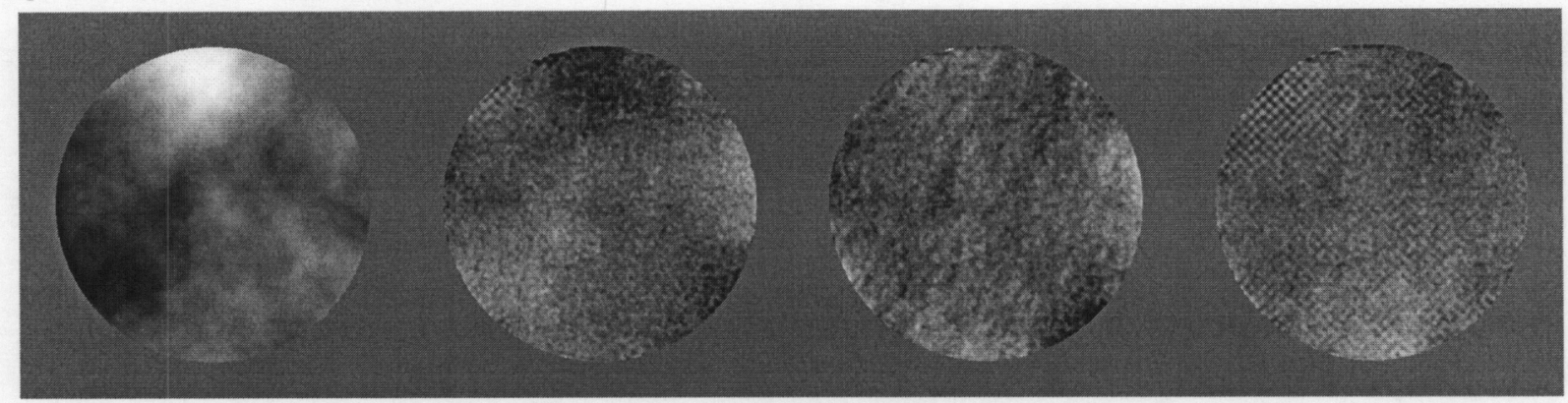

Figure 11. The same WFS measurements as in Figure 10, but this time with the compensated Hudgin-FT and Fried-FT methods. Results for $\lambda=1$ micron. Wavefront and VMM residual are the same. Compensated, filtered Hudgin-FT has mse of 0.340 , Strehl of 0.71. Compensated Fried-FT has mse of 0.311 , Strehl of 0.73.

compensations dramatically improve performance. Compensation and filtering improve Hudgin-FT Strehl in this case from 0.49 to 0.71 . Fried-FT Strehl improves from 0.58 to 0.73 . For comparison, VMM Strehl was 0.66. (All measurements at $\lambda=1$ micron.) Figure 11 shows this error and details the statistics.

\section{CONCLUSIONS}

Analysis of idealized models and Monte Carlo simulations have established that though there are discrepancies between the ideal FT method models and realistic AO systems with SH sensors and a DM, these can in general be compensated for. Sensor gain and misalignment, if measurable, can be compensated for easily at the filtering stage. Furthermore, additional filtering can improve the FT method performance. Low-pass filtering, such as that shown by the non-ideal DM models, can significantly improve both noise propagation and latent error for Fried-FT. The performance of Hudgin-FT can be improved by adding in extra misalignment compensation. Simulations show that in open-loop correction on large systems, FT methods can produce results comparable to or better than VMM methods (derived explicitly from the WFS, DM, gains, misalignment, etc.). Furthermore, this compensation can be done completely at the filtering stage, therefore not increasing the computational burden of reconstruction.

\section{ACKNOWLEDGMENTS}

Thanks to Jim Brase, Don Gavel and Erik Johansson for being so willing to answer my questions. Thanks to Wim deVries for his helpful proof-readings. This work was performed under the auspices of the U.S. Department of Energy by the University of California, Lawrence Livermore National Laboratory under contract No. W-7405Eng-48. The document number is UCRL JC-147058. This work has been supported by the National Science 
Foundation Science and Technology Center for Adaptive Optics, managed by the University of California at Santa Cruz under cooperative agreement No. AST-9876783.

\section{REFERENCES}

1. L. A. Poyneer, D. T. Gavel, and J. M. Brase, "Fast wavefront reconstruction in large adaptive optics systems using the Fourier transform," J. Opt. Soc. Amer. (A), p. in press, 2002.

2. D. L. Fried, "Least-square fitting a wave-front distortion estimate to an array of phase-difference measurements," J. Opt. Soc. Amer. (A) 67, pp. 370-5, 1977.

3. J. Herrmann, "Least-squares wave front errors of minimum norm," J. Opt. Soc. Amer. (A) 70, pp. 28-35, 1980.

4. K. Freischlad and C. Koliopoulos, "Modal estimation of a wave front from difference measurements using the discrete fourier transform," J. Opt. Soc. Amer. (A) 3, pp. 1852-61, 1986.

5. J. V. F. Rigaut and O. Lai, "An analytical model for Shack-Hartmann-based adaptive optics systems," in Adaptive Optical Systems Technoilogies, D. Bonaccini and R. Tyson, eds., Proc. SPIE 3353, pp. 1038-48, 1998.

6. E. M. Johansson and D. T. Gavel, "Simulation of stellar speckle imaging," in Amplitude and Intensity Spatial Interferometry II, J. B. Breckinridge, ed., Proc. SPIE 2200, pp. 372-83, 1994.

7. M. C. Roggemann and B. Welsh, Imaging Through Turbulence, CRC Press, New York, 1996.

8. J. W. Hardy, Adaptive Optics for Astronomical Telescopes, Oxford University Press, New York, 1998.

9. J. M. Brase and et al, "Adaptive optics at Lick Observatory: System architecture and operations," in Adaptive Optics in Astronomy, M. A. Ealey and F. Merkle, eds., Proc. SPIE 2201, pp. 474-88, 1994. 